\title{
Handoff Training for Medical Students: Attitudes, Knowledge, and Sustainability of Skills
}

\author{
Christopher J Smith ${ }^{1}$; Grant Peterson ${ }^{2}$; Gary L Beck ${ }^{3}$ \\ ${ }^{1}$ Department of Internal Medicine, University of Nebraska Medical Center College of Medicine, Omaha, NE, \\ USA. ${ }^{2}$ Internal Medicine, Unity Point Clinic, Ankeny Prairie Trail, Ankeny, IA, USA. ${ }^{3}$ Office of Medical \\ Education, University of Nebraska Medical Center College of Medicine, Omaha, NE, USA.
}

\begin{tabular}{ll}
\hline ARTICLE INFO \\
Received & $: 06 / 03 / 2015$ \\
Accepted & $: 13 / 04 / 2015$ \\
Published & $: 10 / 06 / 2015$
\end{tabular}

\section{KEYWORD}

Handoff

Handover

Care transition

Discharge summary

Medical student

\begin{abstract}
Introduction: The medical community has placed increasing emphasis on ensuring quality patient care handoffs, yet there is relatively little research regarding educational interventions for medical students. Objective: To assess the impact of a handoff communication workshop on senior medical students' attitudes, knowledge, and skills. Method: A 3-hour handoff skills workshop was developed using published research and educational models. The session included didactic lectures, role-play activities, and group discussion. We assessed the workshop's impact using attitudinal, fact-based multiple choice, and case-based questions preceding, immediately following, and several months after the intervention. Result: From 20132014, 59 students participated in the handoff training, with 56 (95\%) completing the pre- and post-tests. Participants' self-reported attitudes improved following the intervention ( $\mathrm{p} \leq .005$ in 12 of 13 questions). The mean number of correct answers on multiple choice questions increased from 6.75 (CI 6.44-7.05) to 8.47 (CI 8.28-8.67) out of 9 (p<.001). Vignette scores improved from 6.00 to 8.14 out of $10(\mathrm{p}<0.001)$. Thirty-six participants $(61 \%)$ completed the pre-, post-, and follow-up vignettes. Postworkshop vignette scores improved significantly (6.28 vs. $8.19, \mathrm{p}<.001)$, but decreased in follow-up testing ( 8.19 vs. 6.97, $\mathrm{p}=.008)$. Conclusion: The handoff workshop improved participants' attitudes, knowledge, and skills, but performance gains declined in the months following training. These findings suggest that handoff training should coincide with the opportunity to apply these skills in a clinical context.
\end{abstract}

(C) Medical Education Department, School of Medical Sciences, Universiti Sains Malaysia. All rights reserved.

CORRESPONDING AUTHOR: Dr Christopher J. Smith, Department of Internal Medicine, University of Nebraska Medical Center College of Medicine, Omaha, NE, USA. Email: csmithj@unmc.edu

\section{Introduction}

Handoffs are exchanges of information that occur when responsibility for patient care is transferred between health professionals (1). This includes a broad range of care transitions, including those within a service line (e.g. at shift change), between units (2) (e.g. from the emergency department to inpatient setting), and between care settings (e.g. discharge from the hospital to ambulatory setting (3)). The Joint Commission reports that communication failures and poor coordination across the care continuum are leading causes of sentinel events (4). Additionally, handoff failures are associated with adverse patient outcomes and inefficient care 
delivery (5-8). Resident work hour restrictions have increased the number of handoffs at teaching hospitals (9), and the Accreditation Council for Graduate Medical Education (10) (ACGME) requires residency programs ensure residents are competent in handoff communication skills.

In the past several years, the undergraduate medical education community has become increasingly interested in handoff training. Medical students commonly witness errors related to handoff communication with resultant negative emotional responses $(11,12)$. According to the Association of American Medical Colleges (AAMC), performing patient handoffs, including at the time of discharge, is a core enstrustable professional activity that all entering residents are expected to perform on day one of residency without direct supervision (13). Despite this, few medical schools have structured handoff or care transitions curricula and direct student involvement in handoffs is inconsistent (14-16).

Published assessments of handoff training interventions aimed at medical students are limited and have typically evaluated participants' self-reported attitudes and confidence, rather than improvements in knowledge or performance $(17,18)$. Studies that tested skills tend to lack pre-intervention data for comparison $(19,20)$. A systematic review of handoff educational interventions noted that most studies failed to provide adequate description of their intervention and that there was a lack of studies examining retention of skills (21), making the optimal timing of handoff training uncertain. To address these issues, we developed a handoff skills workshop and assessed its impact on senior medical students' attitudes, knowledge, and skills immediately following and several months after the intervention.

\section{Method}

\section{Setting}

We offered an elective handoff communication workshop for senior medical students at the University Of Nebraska Medical Center College of Medicine as part of a residency preparatory course. We offered a total of six handoff workshops with 9-12 students per session in February and April of 2013-2014. Prior to the workshop there was no standardized handoff curriculum for medical students at our institution. Students' handoff experiences were typically limited to observations of resident handoffs during their junior-year clerkships. Some senior students may also have performed handoffs as part of their sub-internship rotations.

\section{Workshop description}

The workshop included discussion of handoffs within the hospital (such as at shift change) and at the time of discharge (in the form of discharge summaries). Faculty with expertise in patient care handoffs created the curriculum grounded in principles of adult learning (22). Workshop content was based on published research and practice guidelines. Each session lasted approximately 3 hours. The first 2 hours focused on hospital handoffs and the final hour covered discharge summary creation.

\section{Hospital handoffs}

This portion of the workshop reviewed pertinent research and best practices for hospital handoffs, such as occur when care responsibility is transferred to an overnight team. The learning objectives for the handoff portion of the workshop were to (1) explain how handoffs impact patient safety, (2) list key elements for effective handoff communication, (3) perform a standardized strategy for written and verbal handoff communication, (4) perform "if-then" statements in the context of handoff communication, (5) demonstrate "read-back" strategy in the context of handoff communication, and (6) demonstrate the role of patient prioritization when performing handoffs.

In developing the workshop, we specifically addressed 3 aspects of handoff practice (1) (information transfer, responsibility and accountability, and systems-level elements to facilitate handoff) and followed a similar pedagogical model as described by Darbyshire (18). As illustrated in Table 1, the handoff portion of the workshop combined elements of 
didactic teaching, small-group role-play with skills application, and large group discussions. Handoff skills were practiced via vignette-based role-play scenarios with verbal handoff occurring between student dyads with direct faculty oversight. For verbal handoff, we taught the SAIF-IR strategy, which stands for summary statement, active issues, if-then contingency planning, follow-up activities, interactive questioning, and read-back (23). We chose this strategy because it closely matched the focus of our didactic lesson and emphasized the responsibility of both the giver and recipient of handoff information. Students learned and applied skills in a graduated manner, building on relatively simple skills to develop a more complex and robust skill set. The final activity required learners to review, prioritize, and perform written and verbal handoff for 3 patients of varying complexity. The handoff recipients were then asked to respond to mock nursing phone calls, for which the correct course of action required accurate handoff information.

\section{Discharge summary}

The objectives of the discharge summary portion of the workshop were to (1) describe the purpose of a discharge summary, (2) identify common errors in creating a discharge summary, (3) list the components of an effective discharge summary, and (4) critically evaluate examples of discharge summaries. Table 1 shows the components of the discharge summary portion of the workshop. The majority of the didactic portion focused on addressing common errors in writing discharge summaries, including timeliness of completion, summary length, medication reconciliation, appropriate documentation of functional and cognitive status, and adequately documenting follow-up plans. The discharge summary portion of the workshop concluded with the group reviewing and critically evaluating an example discharge summary.

Table 1: Handoff Workshop Content and Time Requirements, University of Nebraska Medical Center, 20132014

\section{Hospital Handoff Section}

- Introduction (10 minutes):

○ Definition, examples, and challenges

- Group reflection on handoff experiences, including impact on patients

- Role play 1: Student dyads perform written and verbal handoff of challenging clinical vignette prior to instruction

- Group reflection on role play, including identification of barriers

- $\quad$ Didactic (20 minutes):
$\circ \quad$ Functions of handoff
- Impact on patient care (patient-level examples and review of literature)
- Categories of handoff errors and overview of best-practices

- Written handoff (20 minutes)

- Required elements with focus on follow-up tasks and contingency planning

- Role play 2: Learners perform written handoff of clinical vignette (template provided)

- Group reflection on written handoff exercise

- $\quad$ Verbal handoff (20 minutes)
- Required elements and review of SAIF-IR* strategy
- Closed-loop communication using read-back strategy
- Role play 3: Student dyads role-play verbal handoff of vignette using SAIF-IR strategy with direct faculty oversight and peer feedback
- Group reflection on verbal handoff exercise 
- $\quad$ Capstone exercise (45 minutes)

- Review concept of prioritization

○ Role play 3: Each student given 3 patient vignettes and asked to perform prioritized written and verbal handoff

- Group reviews order of prioritization

- Mock nursing phone calls requiring accurate handoff information

\section{Discharge Summary Portion}

- Introduction and group discussion of case example (10 minutes)

- $\quad$ Didactic (20 minutes)

○ Definition and purpose of discharge summary

o Joint Commission requirements

○ Best-practice recommendations

○ Formats: narrative vs. problem-based

○ Common errors and solutions: timeliness, length, medication reconciliation, follow-up plans, discharge condition

- Critical evaluation of example discharge summary (15 minutes)

*SAIF-IR indicates Summary statement, Active issues, If-then contingency planning, Follow-up activities, Interactive questioning, Readback

\section{Assessments}

We designed the workshop assessment based on Kirkpatrick's hierarchy for educational assessment (21). Kirkpatrick's Level 1 (learner reaction) was examined via the institution's standardized evaluation form, which was used for all preparatory course workshops. This 10item survey included 8 statements rated on a Likert scale ( $1=$ strongly disagree to $5=$ strongly agree) and 2 open-ended questions addressing strengths and weakness. To evaluate Kirkpatrick Levels $2 \mathrm{a}$ (attitudes and confidence) and $2 \mathrm{~b}$ (knowledge and skills), we developed preintervention, post- intervention, and follow-up assessments, based the on the expert consensus of the authors. The institutional IRB approved the study as an exempted protocol (\#056-13-EX). Evaluations did not include any identifying information and participation was voluntary. An investigator not involved with workshop instruction or grading assigned participants unique alphanumeric identifiers in order to pair responses. Participants completed the evaluations in the absence of the workshop instructors. Assessment content was pilot tested for clarity prior to distribution with a group of 4 senior medical students not enrolled in the seminar.

\section{Pre/post-intervention assessment}

We conducted pre- and post-intervention evaluations immediately before and after the workshop to measure participants' attitudes, knowledge, and handoff performance (Appendix 1). The first section contained 13 items measuring participants' self-reported attitudes and knowledge regarding handoff communication. These questions were rated on a 5-point Likert scale (strongly disagree=1 to strongly agree $=5$ ). The second section contained 9 knowledge-based multiple choice questions (4 regarding handoffs, 5 regarding discharge summaries). In the final section, we asked participants to review a clinical vignette and write an example of a high-quality verbal handoff dialogue. We listed the SAIF-IR strategy for verbal handoff as reference to ensure that any improvement in scores was related to the workshop, and not just the result of providing a template. Open-ended responses were scored independently by two faculty members based upon a pre-determined 10-point scoring system (Appendix 2) with disagreements settled by consensus.

\section{Follow-up assessment}

We conducted a follow-up survey of participants in June following their workshop to test for retention of knowledge and skills prior to entering residency (Appendix 3). We sent participants a link to an online survey using email addresses they provided during the workshop. The follow-up survey included 5 
knowledge-based multiple-choice questions and a clinical vignette for which participants were asked to write a handoff dialogue in the same manner as the pre/post-assessments. Follow-up multiple-choice questions differed from those on the pre/post-tests to avoid test-retest bias. We scored vignette responses as outlined above. Participants who completed the follow-up survey received a $\$ 10$ gift card.

\section{Analysis}

We compiled scores from all workshops and analyzed them in aggregate as well as by year
(2013 vs. 2014) and month of training (February vs. April). Incomplete surveys were excluded from the analysis. We compared pre/postintervention attitudinal-based responses using Wilcoxon Rank Sum test. For analysis of pre/post-survey multiple choice responses, we used Wilcoxon Sign Test. We analyzed the pre-, post-, and follow-up survey clinical vignette scores using Chi-square testing and Wilcoxon Signed Rank test. We used IBM SPSS Statistics Version 22 (Armonk, NY) for all analyses and considered $\mathrm{p}<.05$ to be statistically significant.

Figure 1: Paired Mean Clinical Vignette Scores over Time for Medical Students Participants in Handoff Workshop, University of Nebraska Medical Center, 2013-2014.

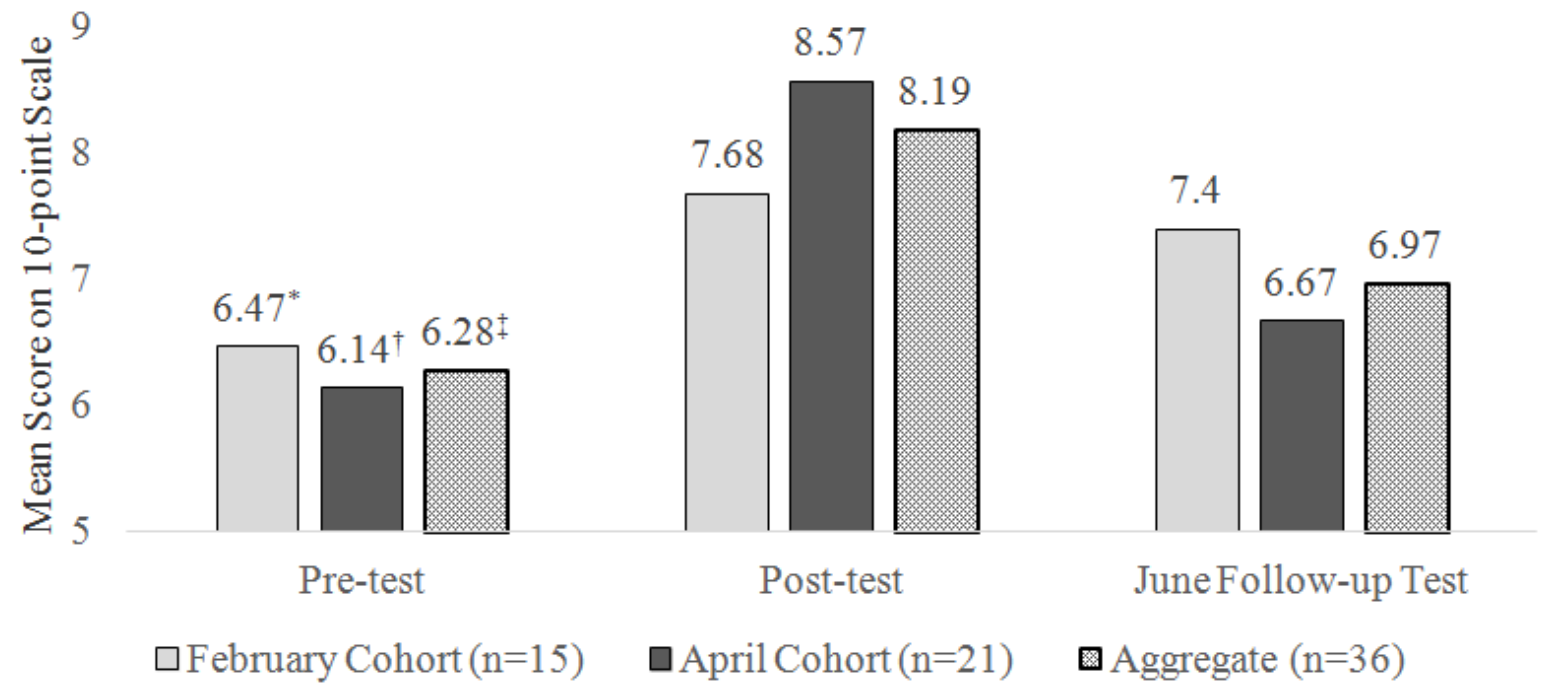

${ }^{*}$ February Cohort, Chi-square $=3.48, \mathrm{p}=0.176 ;{ }^{\dagger}$ April Cohort, Chi-square $=16.33, \mathrm{p}<0.001 ;{ }^{\ddagger}$ Aggregate, Chi-square $=18.82$, $\mathrm{p}<0.001$

\section{Result}

\section{Participant characteristics}

Forty-two percent (25/59) of students were female. Participants were entering 17 different residency disciplines. The most common disciplines were Internal Medicine (14/59, $23.7 \%)$, General Surgery $(8 / 59,13.6 \%)$, and Anesthesiology $(6 / 59,10.2 \%)$. Primary care $(n=22)$ and surgical $(n=21)$ fields each accounted for about one-third of all participants.

\section{Institutional evaluations}

Sixty-eight percent of participants (40/59) completed the institutional evaluation. When compared to the aggregate scores of the other 40 seminars, the handoff workshop received higher average scores for all questions. The largest difference in scores was for the statement "this seminar should be required," for which the handoffs seminar had a combined average of 4.34 on a 5 -point scale, versus 3.55 for other seminars. Overall comments were very positive. Participants praised the interactive nature of the workshop and numerous students stated it was the most valuable workshop they attended.

\section{Pre/Post-intervention assessments}

Fifty-nine of $59(100 \%)$ participants completed the pre- and post-workshop assessments. As 
shown in Table 212 of the 13 questions demonstrated significant improvement addressing participants' attitudes and confidence towards handoffs and discharge summaries $(\mathrm{p} \leq 0.005$ for all). Participants' level of agreement with the statement "handoff communication is important for patient safety" did not change (4.92 vs. $4.92, \mathrm{p}=1.0$ ).

Table 2: Self-reported Attitudes of 59 Medical Students Before and After Handoff Workshop, University of Nebraska College of Medicine, 2013-2014*

\begin{tabular}{|lllcc|}
\hline Handoffs & Pre- & Post- & Z & p \\
\cline { 2 - 6 } 1. I know the critical elements of handoff communication & 2.83 & 4.48 & -6.48 & $<0.001$ \\
2. I can create effective written handoff communication & 3.07 & 4.36 & -6.48 & $<0.001$ \\
3. I know a standardized method for verbal handoff communication & 2.49 & 4.59 & -6.67 & $<0.001$ \\
4. I can effectively give verbal handoff communication & 2.85 & 4.32 & -6.48 & $<0.001$ \\
5. I can effectively receive verbal handoff communication & 3.51 & 4.32 & -5.33 & 0.002 \\
6. I know how to use "read-backs" in verbal handoff communication & 2.66 & 4.63 & -6.35 & $<0.001$ \\
7. I know how to make contingency plans for my patients & 2.64 & 4.25 & -6.45 & $<0.001$ \\
8. I can efficiently handoff patients & 2.48 & 4.09 & -6.52 & $<0.001$ \\
9. I am comfortable providing cross-cover care for patients & 2.24 & 3.56 & -6.31 & $<0.001$ \\
10. Handoff communication is important for patient safety. & 4.92 & 4.92 & .000 & 1.00 \\
\hline Discharge Summaries & & & & \\
1. I know the critical elements of a quality discharge summary & 3.10 & 4.24 & -5.99 & $<0.001$ \\
2. I can create high quality discharge summaries & 2.75 & 3.86 & -5.86 & $<0.001$ \\
3. Discharge summaries are important for patient safety. & 4.68 & 4.92 & -2.80 & 0.005 \\
\hline
\end{tabular}

"Statements rated on 5-point Likert scale, 1=strongly disagree to 5=strongly agree

The mean number of correct multiple choice questions improved from 6.75 (CI 6.44-7.05) to 8.47 (CI 8.28-8.67) (p<.001). Item-level responses analyzed by Wilcoxon Signed-Rank Test showed significant improvement in 4 of 9 multiple-choice questions (Table 3). A question regarding documentation of patients' condition at discharge approached, but did not meet, statistical significance $(\mathrm{p}=.059)$. Mean scores for the open-ended, case-based vignette also significantly improved following the workshop (6.00 to $8.14, \mathrm{p}<.001)$.

Table 3: Paired Multiple-choice Results for 59 Medical Students Participating in Handoff Workshop, University of Nebraska Medical Center, 2013-2014

\begin{tabular}{|c|c|c|c|c|}
\hline Question & $\begin{array}{l}\text { Pre-* } \\
\text { n }(\%)\end{array}$ & $\begin{array}{l}\text { Post-* } \\
\text { n }(\%)\end{array}$ & $\mathbf{z}$ & $\mathbf{p}^{*}$ \\
\hline $\begin{array}{l}\text { 1. Which of the following are critical elements of written handoff content for every } \\
\text { patient? }\end{array}$ & $53(90)$ & $57(97)$ & -1.41 & 0.157 \\
\hline 2. Which of the following are critical elements of verbal handoff? & $25(42)$ & $58(98)$ & -5.75 & $<0.001$ \\
\hline 3. Which of the following is the most effective mode of handoff communication? & $47(80)$ & $59(100)$ & -3.46 & 0.001 \\
\hline $\begin{array}{l}\text { 4. Which of the following is the best strategy to verify handoff information, such as } \\
\text { overnight tasks? }\end{array}$ & $40(68)$ & $57(97)$ & -4.12 & $<0.001$ \\
\hline $\begin{array}{l}\text { 5. Which of the following is a requirement for a discharge summary based upon } \\
\text { Joint Commission guidelines? }\end{array}$ & $56(95)$ & $56(95)$ & 0.00 & 1.00 \\
\hline $\begin{array}{l}\text { 6. What percentage of patients are estimated to have medication errors post- } \\
\text { discharge? }\end{array}$ & $20(43)$ & $47(80)$ & -5.20 & $<0.001$ \\
\hline 7. Important elements of the discharge condition include & $52(88)$ & $57(97)$ & -1.89 & 0.059 \\
\hline 8. Which of the following is a common omission from discharge summaries? & $46(78)$ & $50(85)$ & -1.27 & 0.206 \\
\hline 9. Which of the following is the purpose of a discharge summary? & $59(100)$ & $59(100)$ & 0.00 & 1.00 \\
\hline
\end{tabular}

*Number of students answering correctly. 


\section{Follow-up assessment}

Sixty-eight percent (40/59) of workshop participants completed the follow-up assessment multiple choice questions, with a mean number of correct answers of 4.3 out of 5 . Thirty-six of 59 students (61\%) completed the pre-, post-, and follow-up test vignette question, with significant differences in the paired mean scores (Chisquare $=18.82, \quad \mathrm{p}<.001) . \quad$ Scores improved immediately following the workshop (6.28 vs. $8.19, \mathrm{z}=-4.26, \mathrm{p}<.001$ ), but decreased in June follow-up testing (8.19 vs. 6.97, $\mathrm{z}=-2.65$, $\mathrm{p}=.008)$. The April group scored higher on the post-test than the February group (8.57 vs. 7.68 , $\mathrm{z}=-2.30, \mathrm{p}=.03$ ), but there was no difference in pre-test $(\mathrm{z}=-.224, \mathrm{p}=.823)$ and follow-up test scores $(\mathrm{z}=-.083, \mathrm{p}=.994)$. Vignette scores were not significantly different for the 2013 and 2014 cohorts.

\section{Discussion}

This study demonstrates that an interactive workshop can improve medical students' confidence, knowledge, and skills in performing handoff. This builds on previously published handoff training pedagogy, and is important as handoff education will be expected in undergraduate medical education as part of the new AAMC Core Entrustable Professional Activities for Entering Residency. Indeed, based on participants' feedback this handoff training seminar was expanded in 2014 and will be required of all graduating medical students in 2015.

To our knowledge this is the first study which examined the sustainability of skills following handoff training. Previous medical student handoff curricula have focused on learners' reactions and changes in self-reported attitudes, however, they have typically failed to evaluate higher-level outcomes before and after the intervention. We found that skills, as measured by vignette scores, declined with time, although they remained higher than pre-intervention scores. This decrease may be because learners had limited opportunities to apply their handoff skills in a clinical context after the workshop, as senior medical students have fewer opportunities to take clinically-based elective rotations in the final months of their education. Skill decay is a well-described process which refers to the loss of ability or knowledge after periods of latency. Generally, the longer the period of nonuse of a skill set, the greater the decay (24). Our findings suggest that handoff training for medical students should ideally coincide with the opportunity to apply these skills in a clinical context, such as during a clerkship or subinternship rotations.

This study had several limitations. It was at a single institution, and thus our findings may not be generalizable. As this was an elective workshop, participants may not be representative of all students, although the students demonstrated diverse professional interests. Additionally, our method for assessing verbal handoff skills was not validated and relied on written clinical scenarios. There is ongoing work to create a validated handoff assessment, but it requires direct observation (25). We chose written assessment for practical reasons, in that it allowed us to easily re-test learners via email immediately prior to them entering residency training. To improve the internal validity of the study, two researchers independently scored the vignettes according to a pre-determined scoring system.

Further work is needed in the area of handoff training for medical students. Future studies may include more robust pre- and postintervention assessment of skills, such as in simulation environments. Ultimately, we would like to assess higher-level outcomes, particularly the effect handoff training has on on-the-job behavior (Kirkpatrick Level 3) and patient outcomes (Kirkpatrick Level 4). These studies will be challenging, but there is precedent with resident-level handoff interventions (26).

\section{Conclusion}

Medical students' handoff-related attitudes, knowledge, and skills improved immediately following training, but performance gains declined in the following months. These findings support that handoff training for 
medical students can be effective, but should coincide with assumption of clinical responsibilities.

\section{Acknowledgement}

The authors wish to thank the University of Nebraska Medical Center for providing salary support for Drs. Smith and Beck. We previously presented portions of this data as a poster abstract at the $37^{\text {th }}$ Annual Meeting of the Society of General Internal Medicine.

\section{Reference}

1. Jeffcott SA, Evans SM, Cameron PA, Chin GS, Ibrahim JE. Improving measurement in clinical handover. Qual Saf Health Care. 2009 Aug; 18(4): 272-77.

2. Hilligoss B, Cohen MD. The unappreciated challenges of between-unit handoffs: Negotiating and coordinating across boundaries. Ann Emerg Med. 2013 Feb; 61(2): 155-60.

3. Lenert LA, Sakaguchi FH, Weir CR. Rethinking the discharge summary: A focus on handoff communication. Acad Med. 2014 Mar; 89(3): 393-98.

4. The Joint Commission. Sentinel event data: Root causes by event type 2004-2013 [homepage on the Internet]. 2014 Sep [cited March 5, 2015]. Available from: http://www.jointcommission.org/sentinel_ev ent.aspx.

5. Horwitz LI. Consequences of inadequate sign-out for patient care. Arch Intern Med. 2008; 168(16): 1755-60.

6. Horwitz LI, Meredith T, Schuur JD, Shah NR, Kulkarni RG, Jenq GY. Dropping the baton: A qualitative analysis of failures during the transition from emergency department to inpatient care. Ann Emerg Med. 2009 Jun; 53(6): 701-10.e4.

7. Kitch BT. Handoffs causing patient harm: A survey of medical and surgical house staff. Jt Comm J Qual Patient Saf. 2008; 34(10): 563-70.

8. Ong MS, Coiera E. A systematic review of failures in handoff communication during intrahospital transfers. Jt Comm J Qual Patient Saf. 2011 Jun; 37(6): 274-84.

9. Vidyarthi AR, Arora V, Schnipper JL, Wall SD, Wachter RM. Managing discontinuity in academic medical centers: Strategies for a safe and effective resident sign-out. $J$ Hosp Med. 2006 Jul; 1(4): 257-66.

10. ACGME common program requirements [homepage on the Internet]. 2013 Jul [cited March 5, 2015]. Available from: https://www.acgme.org/acgmeweb/Portals/0/ PFAssets/ProgramRequirements/CPRs2013. pdf.

11. Arora VM, Eastment MC, Bethea ED, Farnan JM, Friedman ES. Participation and experience of third-year medical students in handoffs: Time to sign out? J Gen Intern Med. 2013 Aug; 28(8): 994-98.

12. Koch PE, Simpson D, Toth H, Marcdante K, Densmore E, Young S, Weisgerber $\mathrm{M}$, Morzinski JA, Havas N. Clinical clerkship students' perceptions of (un)safe transitions for every patient. Acad Med. 2014 Mar; 89(3): 477-81.

13. Core entrustable professional activities for entering residency [homepage on the Internet]. . 2014 [cited March 5, 2015]. Available from: https://members.aamc.org/eweb/upload/Core $\%$ 20EPA\%20Faculty\%20and\%20Learner\% 20Guide.pdf.

14. Liston BW, Tartaglia KM, Evans D, Walker C, Torre D. Handoff practices in undergraduate medical education. $J$ Gen Intern Med. 2014 May; 29(5): 765-69.

15. Eskildsen MA, Chakkalakal R, Flacker JM. Use of a virtual classroom in training fourthyear medical students on care transitions. $J$ Hosp Med. 2012 Jan; 7(1): 14-21.

16. O'Toole JK, Stevenson AT, Good BP, Guiot $\mathrm{AB}$, Solan LG, Tse LL, Landrigan CP, Sectish TC, Srivastava R, Starmer AJ, Spector ND, Initiative for Innovation in Pediatric Education-Pediatric Research in Inpatient Settings Accelerating Safe Signouts Study Group. Closing the gap: A needs assessment of medical students and handoff training. J Pediatr. 2013 May; 162(5): 887-8.e1.

17. Chu ES, Reid M, Burden M, Mancini D, Schulz T, Keniston A, Sarcone E, Albert RK. Effectiveness of a course designed to teach handoffs to medical students. J Hosp Med. 2010 Jul-Aug; 5(6): 344-48.

18. Darbyshire D, Gordon M, Baker P. Teaching handover of care to medical students. Clin Teach. 2013 Feb; 10(1): 32-7.

19. Farnan JM, Paro JA, Rodriguez RM, Reddy ST, Horwitz LI, Johnson JK, Arora VM. Hand-off education and evaluation: Piloting the observed simulated hand-off experience 
(OSHE). J Gen Intern Med. 2010 Feb; 25(2): 129-34.

20. Klamen DL, Reynolds KL, Yale B, Aiello M. Students learning handovers in a simulated in-patient unit. Med Educ. 2009 Nov; 43(11): 1097-98.

21. Gordon M, Findley R. Educational interventions to improve handover in health care: A systematic review. Med Educ. 2011 Nov; 45(11): 1081-89.

22. Reed S, Shell R, Kassis K, Tartaglia K, Wallihan R, Smith K, Hurtubise L, Martin B, Ledford C, Bradbury S, Bernstein HH, Mahan JD. Applying adult learning practices in medical education. Curr Probl Pediatr Adolesc Health Care. 2014 Jul; 44(6): 17081.

23. Chu ES, Reid M, Schulz T, Burden M, Mancini D, Ambardekar AV, Keniston A, Albert RK. A structured handoff program for interns. Acad Med. 2009 Mar; 84(3): 347-52.
24. Arthur W, Bennett W, Stanush P.L., McNelley TL. Factors that influence decay and retention: A qualatative review and anlysis. Human Performance. 1998; 11(1): 57-101.

25. Arora VM, Berhie S, Horwitz LI, Saathoff M, Staisiunas P, Farnan JM. Using standardized videos to validate a measure of handoff quality: The handoff mini-clinical examination exercise. J Hosp Med. 2014 Mar 24.

26. Starmer AJ, Sectish TC, Simon DW, Keohane C, McSweeney ME, Chung EY, Yoon CS, Lipsitz SR, Wassner AJ, Harper MB, Landrigan CP. Rates of medical errors and preventable adverse events among hospitalized children following implementation of a resident handoff bundle. JAMA. 2013 Dec 4; 310(21): 2262-70.

Appendix 1: Handoff Workshop Pre/Post-intervention Survey

Please indicate your planned specialty area (e.g. Urology):

Please indicate your level of agreement or disagreement with the following statements using the scale provided: $1=$ Strongly Disagree, 2=Disagree, 3=Neither Agree nor Disagree, 4=Agree, 5=Strongly Agree

Handoffs:

1. I know the critical elements of handoff communication

2. I can create effective written handoff communication

3. I know a standardized method for verbal handoff communication

4. I can effectively give verbal handoff communication

5. I can effectively receive verbal handoff communication

6. I know how to use "read-backs" in verbal handoff communication

7. I know how to make contingency plans for my patients

8. I can efficiently handoff patients

9. I am comfortable providing cross-cover care for patients

10. Handoff communication is important for patient safety.

\section{Discharge Summaries:}

1. I know the critical elements of a quality discharge summary

2. I can create high quality discharge summaries

3. Discharge summaries are important for patient safety.

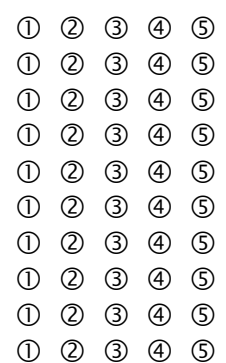

(1) (2) (3) (4) (5)

(1) (2) (3) (4) (5)

(1) (2) (3) (4) (5)

Choose the one best answer for each of the questions below.

1. Which of the following are critical elements of written handoff content for every patient?
a. Recent or planned procedures
b. Allergies
c. Day-by-day recap of hospital course
d. Most recent labs
e. Both A \& B

2. Which of the following are critical elements of verbal handoff?
a. Sickest patients discussed last
b. Opportunity to ask questions
c. Reviewing all comorbidities
d. Detailed review of admission $\mathrm{H} \& \mathrm{P}$
e. Both A \& B 
3. Which of the following is the most effective mode of handoff communication?

a. Phone conversation with electronic handoff template

b. Recorded message

c. Face-to-face

d. Face-to-face with electronic handoff template

e. Recorded message with emailed written handoff

f. Email written handoff

4. Which of the following is the best strategy to verify handoff information, such as overnight tasks?
a. Using a check-list
b. Read-back
c. Non-verbal acknowledgment (e.g. nodding)
d. Verbal acknowledgment (e.g. saying "okay")
e. Written acknowledgement (e.g. signing-off)

5. Which of the following is a requirement for a discharge summary based upon Joint Commission guidelines?
a. Reason for hospitalization
b. Detailed physical exam
c. Daily lab values
d. A $\log$ of medications administered in the hospital
e. Consultation opinions

6. What percentage of patients are estimated to have medication errors post-discharge?
a. $15 \%$
b. $5 \%$
c. $80 \%$
d. $50 \%$

7. Important elements of the discharge condition include
a. Cognitive status
b. Mobility description
c. ADL description if appropriate
d. Fall risk estimation
e. All of the above

8. Which of the following is a common omission from discharge summaries?
a. Medications administered in the hospital
b. Consultations ordered
c. Pending tests at discharge
d. Date of discharge

9. Which of the following is the purpose of a discharge summary?

a. Creating more work for interns

b. Creating a billable document serving as a progress note

c. Creating an overview of the patient's hospital stay from admission to dismissal which can be conveyed to future healthcare providers

d. Creating a medication list the patient can use for future care and prescriptions

e. Creating a detailed, day-to-day summary of the patient's hospital course.

10. You are an intern on a Family Medicine service providing handoff of the following patient to the night float team. Based on the clinical vignette, write a dialogue that might occur during a high-quality verbal handoff between yourself and the overnight resident. You may use any standardized communication strategy, such as the "SAIFIR" model (Summary, Active Issues, If-then contingency planning, Follow-up tasks, Interactive questions, Readback).

Mrs. X is a 79 year old woman admitted 4 days ago with pneumonia. She was initially hypoxic, requiring $3 \mathrm{~L}$ of $\mathrm{O} 2$ by nasal cannula. With antibiotic treatment (ceftriaxone and azithromycin, day 4 of 10), she has improved and is currently doing well on ambient air. Her other vital signs are within normal limits. Mrs. X is a former kindergarten teacher. She is on a general diet. On admission, she also had acute renal failure with a creatinine of $1.6 \mathrm{mg} / \mathrm{dL}$ (baseline $0.8 \mathrm{mg} / \mathrm{dL}$ ). This has improved, but she has a BMP ordered for 10:00 pm tonight. If her creatinine is above 1.2, you want her to receive $1 \mathrm{~L}$ of normal saline. Two nights ago she received lorazapam to aid with sleep and became delirious, so benzodiazepines should be avoided. Her other chronic medical problems include well-controlled hypertension, overactive bladder, and skin tags. You expect she will discharge home in 1-2 days. 
Appendix 2: Verbal Handoff Vignette Scoring System

One point given for each of the following elements with 10 points possible:

Pre/post-test:

- Use of standardized format and logical flow of handoff communication

- Brief summary of case

- Description of current clinical condition (e.g. pertinent vital signs and exam findings)

- Mentions pneumonia and treatment

- Mentions acute renal failure

- $\quad$ Follow-up laboratory testing with follow-up plan

- $\quad$ Contingency plan with instructions to avoid benzodiazepines

- $\quad$ Handoff receiver questions

- Handoff receiver read-back for follow-up laboratory testing

- $\quad$ Handoff receiver read-back for avoiding benzodiazepines

- Deduct one point for irrelevant data - kindergarten teacher, diet, non-active chronic medical conditions

Follow-up Test:

- Use of standardized format and logical flow of handoff communication

- $\quad$ Brief summary of case

- Description of current clinical condition (e.g. pertinent vital signs and exam findings)

- Recent change in clinical status with likely pneumonia

- Mention of full code status

- $\quad$ Follow-up chest x-ray with follow-up plan

- $\quad$ Contingency plans for worsening hypoxia

- Handoff receiver questions

- Handoff receiver read-back for follow-up chest x-ray

- Handoff receiver read-back for worsening hypoxia.

- $\quad$ Deduct one point for irrelevant data - family history of arthritis, hyperlipidemia

\section{Appendix 3: Patient Handoff Seminar Post-test}

Choose the one best answer for each of the questions below.

1. Which of the following is the purpose of a discharge summary?

a. Creating a detailed, day-to-day summary of the patient's hospital course.

b. Creating a billable document serving as a progress note

c. Creating an overview of the patient's hospital stay from admission to dismissal which can be conveyed to future healthcare providers

d. Creating more work for interns

e. Creating a medication list the patient can use for future care and prescriptions

2. A patient was discharged 3 days ago from the hospital and is now in your clinic for follow-up. He was treated for a pulmonary embolism with a subcutaneous blood thinner as a bridge to oral warfarin. Which of the following is a component of the discharge summary that would aid the most in providing further care for this patient?

a. A description of the admission physical exam, including lung auscultatory findings

b. A list of pending labs at the time of discharge

c. Details of oral potassium replacement

d. A complete copy of the radiologist's chest CT report

e. Review of vital signs on the day of discharge

3. You are creating a discharge summary for a patient who had a heart failure exacerbation. She has recovered after a change in their daily diuretic regimen. Which of the following would you include to help the primary provider?
a. An updated medication list.
b. A listing of the patient's ideal "dry" weight
c. Documentation of the patient's night-time confusion
d. Both a. and c.
e. All of the above

4. You are creating a discharge summary for a patient who had complications from a partial colectomy performed to resect colon cancer. The patient is leaving for a rehab facility after a 10 day hospitalization. Which of the following is the most important to include in the discharge summary?
a. "On day 7 the patient had an abdominal x-ray, which showed normal bowel gas pattern."
b. "The patient requires a two person assist when transferring from bed."
c. "On day 4 patient's diet was advanced from clears to full liquids."
d. "The patient received a total of 6 L of IV NS and maintained good urine output." 
e. "The patient's chronic hypertension remained stable throughout the hospitalization."

5. When handing-off a patient to a cross-cover resident, which of the following is true about the order in which you should discuss patients

a. Sicker patients should be discussed last

b. Patients should be discussed based on their length of stay in the hospital, as people with longer stays will require more time to during handoff communication

c. Discharging patients should be discussed first

d. Sicker patients should be discussed first

e. Patients should be discussed based on their geographic location in the hospital (ward-based handoff)

Vignette: You are an intern on a Family Medicine service giving handoff to the night float team. Based on the clinical vignette, write a dialogue that might occur during a high-quality verbal handoff between yourself and the overnight resident. Use the "SAIF-IR" model (Summary, Active Issues, If-then contingency planning, Follow-up tasks and plan, Interactive questions, Read-back).

Mrs. X is a 79 year old woman admitted 4 days ago with delirium from a urinary tract infection. She has a family history of rheumatoid arthritis and is a full code. She is on ciprofloxacin for her UTI and her delirium has resolved, but one hour ago she developed new onset hypoxia requiring $4 \mathrm{~L}$ of $\mathrm{O} 2$ per nasal cannula to keep her O2 saturations above $90 \%$. Her other vital signs are temperature $38^{\circ} \mathrm{C}$, heart rate 98 , respiratory rate 16 , blood pressure $110 / 75$. Her past medical history includes hyperlipidemia. She is breathing comfortably without accessory muscle use and has right lower lobe rales. You suspect pneumonia. A chest $\mathrm{x}$-ray is pending, which you want the overnight team to evaluate. If there is a consolidation, you want piperacillin started. If her hypoxia worsens to the point that she requires more than $6 \mathrm{~L}$ nasal cannula, she should be sent to the ICU. 